Case report

\title{
Portal cavernoma in a case of alcoholic liver disease with myeloproliferative neoplasm
}

\author{
Xingshun Qi, Chunyan Wu, Yue Hou, Xiaozhong Guo \\ Liver Cirrhosis Study Group, Department of Gastroenterology, General Hospital of Shenyang Military Area, Shenyang 110840, China
}

\begin{abstract}
Chronic liver disease is a major cause of portal vein thrombosis. Further screening for other thrombotic risk factors in patients with portal vein thrombosis and chronic liver diseases is often neglected. In this paper, we report a case of portal cavernoma in a patient who was diagnosed with alcoholic liver disease and myeloproliferative neoplasm.
\end{abstract}

Key words: portal vein thrombosis, liver, risk factor, hematology, spleen.

\section{Address for correspondence}

Prof. Xiaozhong Guo, Dr. Xingshun Qi, Department of Gastroenterology, General Hospital of Shenyang Military Area, No. 83 Wenhua Road, Shenyang, 110840 China, tel. 86-24-28897603, fax 86-24-28851113, e-mail: guo_xiao_zhong@126.com (XG), xingshunqi@126.com (XQ)

\section{Introduction}

Chronic liver disease, especially liver cirrhosis, is one of the most common causes of portal vein thrombosis (PVT) $[1,2]$. Once chronic liver diseases are diagnosed in patients with PVT, further screening for other thrombotic risk factors is often neglected. Herein, we report that portal cavernoma was diagnosed in a case of alcoholic liver disease with myeloproliferative neoplasm (MPN).

\section{Case report}

A 76-year-old man was admitted to the Department of Gastroenterology of the General Hospital of Shenyang Military Area due to persistent abdominal discomfort and intermittent melena for 2 weeks. He had drunk $100 \mathrm{~g}$ of white wine every day for about 30 years, but abstained from alcohol drinking 8 years ago. He did not have any history of viral hepatitis. On physical examinations, the shifting dullness was suspicious. On laboratory tests, white blood cell (WBC) count was $21.2 \times 10^{9} / 1$ (reference range [RR]: $4-10 \times 10^{9} / 1$ ), percentage of neutrophils was $88.4 \%$ (RR: $50-70 \%$ ), red blood cell count was $4.51 \times 10^{12} / \mathrm{l}\left(\mathrm{RR}: 4-5.5 \times 10^{12} / \mathrm{l}\right)$, hemoglobin was $76 \mathrm{~g} / \mathrm{l}$ (RR: $110-170 \mathrm{~g} / \mathrm{l})$, platelet count (PLT) was $265 \times 10^{9} / 1$ (RR: $\left.100-300 \times 10^{9} / 1\right)$, alkaline phosphatase was $108 \mathrm{U} / \mathrm{l}$ (RR: 45-125 U/l), $\gamma$-glutamyl transpeptidase was $27 \mathrm{U} / \mathrm{l}$ (RR: 10-60 U/l), total bilirubin was $11.9 \mu \mathrm{mol} / \mathrm{l}$ (RR: 5.1-22.2 $\mu \mathrm{mol} / \mathrm{l}$ ), albumin was $37.6 \mathrm{~g} / \mathrm{l}$ (RR: 40-55 g/l), serum creatinine was $97.0 \mu \mathrm{mol} / \mathrm{l}$ (RR: $44-133 \mu \mathrm{mol} / \mathrm{l}$ ), and the international normalized ratio was 1.26. Abdominal contrast-enhanced computed tomography scans demonstrated portal cavernoma, intrahepatic bile duct dilation, splenomegaly, splenic artery aneurysm with thrombosis, and mild ascites (Fig. 1). In detail, a normal main portal vein in the hepatic hilum was replaced by collateral vessels, which was called cavernous transformation of the portal vein (CTPV) or portal cavernoma. Intrahepatic bile duct dilation should be portal biliopathy in this case, which was primarily caused by compression of the bile duct by collateral vessels. Given that the liver surface was relatively smooth, transient elastography was further performed and demonstrated a liver stiffness value of $13.7 \mathrm{kPa}$. Thus, he was diagnosed with alcoholic liver disease and portal cavernoma. The ChildPugh score was 6 points.

On the other hand, in spite of splenomegaly, PLT was close to the upper limit of the normal range, and 

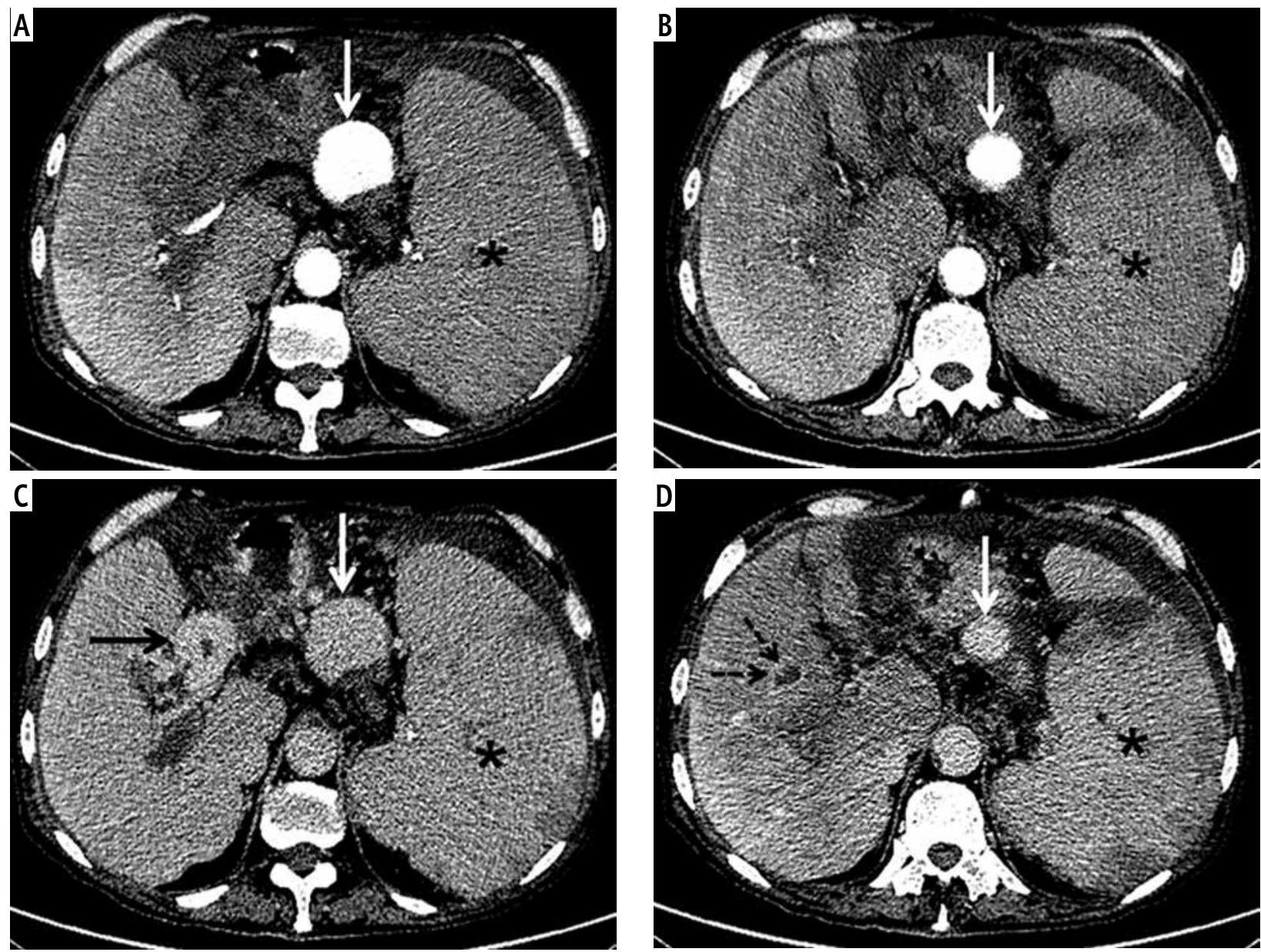

Fig. 1. Abdominal contrast-enhanced computed tomography (CECT) scans. A-B) Arterial phase of CECT, C-D) portal venous phase of CECT. Asterisk represents the splenomegaly; white arrows represent the splenic artery aneurysm with thrombosis; black solid arrows represent the portal cavernoma; and black dotted arrows represent the intrahepatic bile duct dilation

WBC was far beyond the upper limit of the normal range. JAK2 V617F mutation was positive. Bone marrow biopsy confirmed a diagnosis of MPN. He was transferred to the Department of Hematology. Both antinuclear antibody and anti-double-stranded DNA antibody were negative. Hemolytic tests, including erythrocyte osmotic fragility, heat hemolysis test, sucrose test, Ham's test, and Coombs' test AHG, IgG, $\mathrm{C} 3 \mathrm{~d}$, and Ctrl, were negative. $\operatorname{IgA}, \operatorname{IgG}, \operatorname{IgM}, \mathrm{C} 3$, and $\mathrm{C} 4$ levels were within the normal range. Hydroxyurea was prescribed. Symptomatic treatment was given for the management of ascites. Anticoagulants were not given due to the absence of de novo portal vein thrombosis and presence of CTPV. Then, he was discharged and was closely followed.

\section{Discussion}

Until now, the role of other concomitant predisposing factors in the development of PVT in patients with chronic liver diseases remains unclear. Ph-negative MPN is the most common cause of idiopathic PVT [3]. Currently, screening for JAK2 V617F mutation is the first step for diagnosing MPN [4]. Our previous metaanalysis demonstrated that the JAK2 V617F mutation was positive in $24 \%$ of non-malignant and non-cirrhotic patients with PVT; by comparison, the JAK2 V617F mutation was positive in only $7 \%$ of cirrhotic patients with PVT [5]. Our prospective observational study also found that the JAK2 V617F mutation was positive in only $1.4 \%(1 / 71)$ of cirrhotic patients with PVT [6]. Thus, MPN is rarely observed in cirrhotic patients with PVT, and screening for MPN is also very limited in such patients. In our case, the most important clue for the diagnosis of MPN was the presence of splenomegaly but a relatively high PLT. Indeed, in patients with chronic liver disease, PLT is often decreased and/or below the lower limit of the normal range because of hypersplenism and splenomegaly; by comparison, in patients with MPN, PLT is always increased. 
In conclusion, this case suggested the possibility that concomitant MPN contributes to the development of PVT in cases of chronic liver diseases. Myeloproliferative neoplasm should be thoroughly checked once the conditions of trilineage hematopoiesis have been found to be inconsistent with the change of regular blood tests in chronic liver diseases.

\section{Disclosure}

Authors report no conflict of interest.

\section{References}

1. Qi X, Han G, Fan D. Management of portal vein thrombosis in liver cirrhosis. Nat Rev Gastroenterol Hepatol 2014; 11: 435-446.

2. Qi X, Li H, Liu X, et al. Novel insights into the development of portal vein thrombosis in cirrhosis patients. Expert Rev Gastroenterol Hepatol 2015; 9: 1421-1432.

3. De Stefano V, Qi X, Betti S, et al. Splanchnic vein thrombosis and myeloproliferative neoplasms: molecular-driven diagnosis and long-term treatment. Thromb Haemost 2016; 115: 240-249.

4. Vardiman JW, Thiele J, Arber DA, et al. The 2008 revision of the World Health Organization (WHO) classification of myeloid neoplasms and acute leukemia: rationale and important changes. Blood 2009; 114: 937-951.

5. Qi X, Yang Z, Bai M, et al. Meta-analysis: the significance of screening for JAK2V617F mutation in Budd-Chiari syndrome and portal venous system thrombosis. Aliment Pharmacol Ther 2011; 33: 1087-1103.

6. Qi X, Zhang C, Han G, et al. Prevalence of the JAK2V617F mutation in Chinese patients with Budd-Chiari syndrome and portal vein thrombosis: a prospective study. J Gastroenterol Hepatol 2012; 27: 1036-1043. 